\title{
Smooth muscle cells and the pathogenesis of the lesions of atherosclerosis
}

\author{
Elaine W Raines, Russell Ross
}

The size, elasticity, and integrity of the large arteries is determined by the smooth muscle cells (SMCs) of the tunica media and by the connective tissue matrix including collagen, elastin, and proteoglycans, synthesised and deposited by the SMCs. The central cellular feature of the lesions of atherosclerosis is the accumulation of SMCs, together with macrophages and lymphocytes, in the tunica intima of these arteries. SMCs are present in various amounts in early fatty streaks, in which macrophages are the principal cell type, and then become dominant as the streaks progress to fibrofatty lesions. ${ }^{1}$ In fibrous plaques, however, SMCs are the predominant cell type and their accumulation and proliferation are critical in determining the extent and characteristics of these advanced lesions and whether they will lead to clinical sequelae. In addition to their capacity to synthesise large amounts of connective tissue, SMCs can accumulate lipid. Their metabolism seems to be altered in the developing lesions of atherosclerosis and, as a consequence, their metabolic products may further contribute to lesion progression. Attempts to determine the mechanisms of lesion formation and progression must account for the multiplicity of initiating factors, the focal nature of the lesions, the accumulation and proliferation of smooth muscle cells within the intima, the formation of connective tissue, and the accumulation of lipid by the extracellular matrix and by these cells.

In this overview we summarise the changes seen in SMCs associated with lesions of atherosclerosis and discuss the known regulators of SMC migration, proliferation, and metabolism that may contribute to the development of the lesions of atherosclerosis. We will not discuss the different hypotheses on the pathogenesis of atherosclerosis that are extensively covered in other reviews. ${ }^{2-6}$ Rather, we will focus our comments on smooth muscle cell biology and emerging concepts of the regulation of smooth muscle cell function in vascular disease.

\footnotetext{
Department of Pathology, University of Washington, Seattle, Washington, USA

E W Raines

R Ross

Correspondence to Dr E W Raines, Department of Pathology, School of Medicine, University of Washington, Seattle Washington 98195, USA
}

\section{Changes in smooth muscle cells} associated with lesions of atherosclerosis MIGRATION INTO THE INTIMA

Evaluation of the contribution of migration to the human disease is difficult because at least some of the lesions may form in pre-existing collections of intimal SMCs called cushions or intimal masses. ${ }^{1}$ Experimental studies after balloon endothelial denudation indicate that the neointimal SMCs are derived from the media. The expanded balloon leads to platelet adherence and release and SMC injury both of which lead to early replication of SMCs in the media. This is followed by migration into the intima and further replication. ${ }^{7}$ Similarlyo after cholesterol-induced injury, morphologi cal evidence and studies of tritiated thymidines incorporation are consistent with recruitmentof both intimal and medial cells into thes growth fraction, but the accumulation of cells is limited to the intima. The same sequence of events probably occurs in humans, thougkf complicated by the diffuse intimal thickenings that may also contribute to lesion growth.

\section{PROLIFERATION IN THE INTIMA AND UPPER} MEDIA

In many cases clinically significant stenoses may develop over several decades and prote bably represent a complex biological response to chronic injury that has been characterised in hypercholesterolaemic animals. ${ }^{2}$ Angio graphic studies in humans showed thae established plaques can remain unchanged over several years of observation. ${ }^{8}$ Therefore it is not surprising that analyses of SMC proe liferation in human atherosclerotic lesions using antibodies to cell cycle-related protein estimate replication rates of less than $1 \%$. Though a few plaques had higher proliferative indices, the observed low level of replication may reflect the variability in the growth rates of plaques observed in the angiographic studi ies. Such observations are also consistent with the possibility that the growth of plaques may be episodic, particularly in coronary lesions? where the contribution of fissuring ant thrombosis to plaque progression is becoming increasingly clear. ${ }^{5}$ Alternatively, the low proo liferative level may accord with a greater role for the migration of cells from the media inte the intima. Analysis of the proliferative activie ty in lesions after repeat coronary atherectom indicate that the proliferative rate seems to bळ much higher if the restenotic tissue is removed less than six months after atherecto $\frac{0}{\overline{0}}$ my, ${ }^{10}$ suggesting that restenotic lesions are़ more active and are perhaps younger lesions. $\frac{\stackrel{\Omega}{\mathbb{D}}}{\mathrm{O}}$
PHENOTYPIC MODULATION OF SMOOTH MUSCLE CELLS

Normally, adult medial SMCs (terme "contractile") are arranged in concentrio layers and are filled with myofilaments an dense bodies but contain a relatively poorly developed Golgi apparatus and rough endoplasmic reticulum. In contrast, SMCs 
characteristic of the intimal lesions of atherosclerosis (termed "synthetic") have lost this appearance and are characterised by an abundance of rough endoplasmic reticulum and Golgi bodies with few and sometime no evident myofilaments. ${ }^{112}$ Similar SMC morphology is observed in the vessels during embryonic development and in the neointima that develops after the induction of intimal lesions by balloon catheterisation.

Because of the complexity of the in vivo environment, cell culture studies have been used to explore and define further these two different phenotypes as well as the factors that mediate these changes. ${ }^{112}$ Unlike freshly isolated medial cells, which are contractile, cultured cells lose their contractility and myofilaments and develop an extensive rough endoplasmic reticulum and a large Golgi complex that parallel their activities in the synthesis and secretion of extracellular matrix components. At the same time there is a reduced expression of $a$-actin mRNA, the cells express genes for growth factors and their receptors, and, in response to exogenous stimulation, they divide. However, modulation into this synthetic state in vitro can be inhibited by growing the SMCs on a confluent layer of endothelial cells and by particular vasoactive agents and extracellular matrix constituents (see below).

ACCUMULATION AND OXIDATION OF LIPID

Many epidemiological studies have shown an association between chronically raised concentrations of plasma low density lipoproteins and increased incidence of atherosclerosis. Recently, several clinical trials have shown that considerable lowering of plasma cholesterol can result in angiographically demonstrable, quantitatively significant regression of lesions. ${ }^{8}$ Both macrophages and SMCs can accumulate lipid and have been identified as foam cells within lesions of atherosclerosis. ${ }^{2}$ Though SMCs, like macrophages, are capable of modifying lipoproteins by oxidation to increase their atherogenicity, ${ }^{4}$ oxidised low density lipoprotein epitopes are rarely found associated with areas that are rich in smooth SMCs. In vivo studies in Watanabe heritable hyperlipidaemic (WHHL) rabbits (an animal model for familial hypercholesterolaemia) implicate oxidatively modified LDL in the development of atherosclerosis. Administration of probucol, a powerful antioxidant, was associated with a dramatic reduction in the rate of development of atherosclerosis and resulted in smaller, less cellular lesions. Interestingly, SMCs were the predominant cell type in lesions from probucol-treated animals, whereas macrophages predominated in lesions from controls. ${ }^{13}$

\section{SYNTHESIS OF MATRIX COMPONENTS}

Extracellular matrix accumulates within the lesions as the disease progresses and SMCs are solely responsible for its synthesis. Collagens constitute a major class of protein within arteries, and type I collagen (together with lesser amounts of types III, IV, and V) accumulates during the formation of the plaque. ${ }^{14}$ The increased deposition of collagen in the lesions alters the mechanical properties of the wall, making it more susceptible to changes in the flow characteristics of the blood and more susceptible to fissuring or ulceration or both as well as altering the vascular resistance at some sites.

All of the major families of proteoglycans also accumulate in lesions during their development. ${ }^{14}$ Proteoglycans are able to bind several molecules involved in lipid accumulation, calcification, and thrombosis: consequently, their presence may further predispose the lesions to these events. Proteoglycans have also been implicated in growth control within the artery wall. Most notable, heparan sulphates derived from heparan sulphate proteoglycans synthesised by endothelial cells and SMCs are potent inhibitors of SMC growth. ${ }^{15}$

\section{A central role for growth regulatory molecules in altered SMC activity}

CYTOKINES AND GROWTH FACTORS: TIGHTLY REGULATED LOCAL MEDIATORS

Cytokines and growth factors constitute a potent set of multifunctional peptide signalling molecules capable of regulating several cellular functions, including chemotaxis or directed migration, proliferation, accumulation of lipid, and synthesis of matrix components - all of which take place during atherogenesis. "Cytokine" is the term that has been used to describe the family of peptides that regulate immune function and the term "growth factor" has most often been applied to stimulators and inhibitors of cell proliferation. These growth regulatory molecules are multifunctional and a single peptide can promote cellular changes at several different levels. For example, platelet-derived growth factor (PDGF) induces directed migration and proliferation of SMCs but also increases uptake of low density lipoprotein and synthesis of matrix proteins and stimulates vasoconstriction. ${ }^{16}$ However, the action of cytokines and growth factors is limited to cells that express specific surface receptors. The actions of many growth-regulatory molecules may be limited to one or two cell types. For example, PDGF receptors are present on SMCs but not on arterial endothelial cells. In addition, receptor expression may be low in normal medial SMCs and become upregulated after exposure to stimuli such as injurious agents, as have been demonstrated for PDGF receptors.

Both the growth-regulatory molecules and their receptors are tightly but independently regulated. Growth factors and cytokines can be synthesised by resident cells in the artery wall as well as by infiltrating cells from the circulation. The table (A) lists the growth factors and cytokines known to alter SMC function and their cellular sources. ${ }^{1718}$ If we consider the growth factors capable of inducing smooth muscle cell proliferation (PDGF, IGF-I, IL-1, and TNF- $\alpha$ ), they are expressed 
Stimulants and inhibitors of smooth muscle cell migration, proliferation, and phenotype

\begin{tabular}{|c|c|c|c|c|}
\hline \multirow[b]{2}{*}{ Substance } & \multirow[b]{2}{*}{ Cellular sources * } & \multicolumn{3}{|c|}{ Effect on smooth muscle } \\
\hline & & Proliferationt & Migrationt & Phenotypef \\
\hline \multicolumn{5}{|c|}{ (A) Growth factors and cytokines } \\
\hline Epidermal growth factor (EGF) & $\mathrm{P}$ & + & & \\
\hline Basic fibroblast growth factor (bFGF) & $\mathrm{EC}, \mathrm{M}, \mathrm{SMC}$ & ++ & 0 & \\
\hline Heparin-binding EGF-like growth factor (HB-EGF) & EC, M, SMC & ++ & + & \\
\hline Insulin growth factor-I (IGF-I) & $\mathrm{EC}, \mathrm{M}, \mathrm{P}, \mathrm{SMC}$ & + & + & \\
\hline Interferon- $-\gamma^{\prime}\left(\right.$ IFN- $\left.-\eta^{\prime}\right)$ & $\mathrm{M}, \mathrm{T}$ & \pm & & \\
\hline Interleukin-1 (IL-1) & EC, $M, P, S M C, T$ & + & & \\
\hline Interleukin-6 (IL-6) & EC, M, SMC, T & + & & て \\
\hline Platelet-derived growth factor (PDGF) & EC, M, P, SMC & + & + & \\
\hline Thrombin & P1 & + & & \\
\hline Transforming growth factor $\alpha$ (TGF- $\alpha$ ) & M & + & & \\
\hline Transforming growth factor $-\beta$ (TGF- $\beta$ ) & EC, M, P, SMC, T & \pm & + & \\
\hline Tumour necrosis factor- $a$ (TNF- $a$ ) & EC, M, SMC, T & + & & \\
\hline \multicolumn{5}{|c|}{ (B) Vasoactive agents, prostaglandins, and leukotrienes } \\
\hline Angiotensin II (A-II) & $\mathrm{EC}, \mathrm{P} 1, \mathrm{SMC}$ & +8 & & \\
\hline Atrial natriuretic polypeptide (ANP) & $\mathrm{CM}$ & - & & \\
\hline $\begin{array}{l}\text { Endothelial-derived relaxing factor-nitric oxide } \\
\text { (EDRF-NO) }\end{array}$ & EC, M, NP, SMC & - & & \\
\hline Endothelin (ET-1) & $\mathrm{EC}, \mathrm{M}, \mathrm{P}, \mathrm{Pl}$ & + & & \\
\hline Adrenaline/noradrenaline & AH & + & & \\
\hline 12-hydroxyeicosatetraenoic acid (12-HETE) & $\mathrm{P}$ & & + & \\
\hline Leukotriene $\mathrm{B}_{4}\left(\mathrm{LTB}_{4}\right)$ & $\mathrm{N}$ & & + & \\
\hline Prostacyclin $\left(\mathrm{PGI}_{2}\right)$ & EC, SMC & - & & \\
\hline Prostaglandin $\mathrm{E}_{1}\left(\mathrm{PGE}_{1}\right)$ & EC, SMC & - & & $\mathrm{S}$ \\
\hline Serotonin & $\mathrm{P}$ & +8 & & 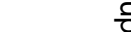 \\
\hline Substance $\mathrm{P}$ & NP & + & & c \\
\hline Vasopressin & NP & $+s$ & & \\
\hline \multicolumn{5}{|c|}{ (C) Extracellular matrix } \\
\hline Fibronectin (FN) & EC, M, P, Pl, SMC & & + & $\mathrm{S}$ \\
\hline Heparin & EC, SMC & - & - & \\
\hline Laminin & EC, SMC & & & C \\
\hline Osteonectin (SPARC) & P, EC, SMC & & - & 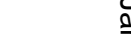 \\
\hline Tenascin $(\mathrm{TN})$ & SMC & & & \\
\hline Thrombospondin (TSP) & P, EC, SMC & + & & 0 \\
\hline
\end{tabular}

*Key to cellular sources: $\mathrm{AH}$, adrenal hormone; CM, cardiac myocyte; EC, endothelial cell; M, monocyte/macrophage; $\mathrm{N}_{2}$ neutrophil; NP, neuropeptide; P, platelet; P1, plasma; SMC, smooth muscle cell; SPARC, secreted protein acidic and rich ino

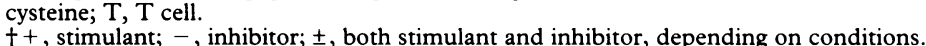

$\dagger$
$\ddagger S$, stimulant;
- , inhibicitor; $C$, contractile.

§Stimulation of cell hypertrophy, but not cell proliferation.

at low or undetectable concentrations in normal human arteries but are increased after injury or in atherosclerotic lesions. ${ }^{18}$ bFGF, however, is ubiquitously detected in the basement membranes of blood vessels of all sizes and within smooth muscle cells of the tunica media. Because bFGF is not secreted by smooth muscle cells and basement membrane components are known to bind bFGF, basement membranes may represent a storage pool of bFGF and its release may be induced by cellular injury or by enzymes such as neutrophil and mast cell heparinases. Therefore, in the normal artery, growth factors and cytokines are either expressed at low or undetectable concentrations or sequestered in storage pools. In both cases, activation or injury is required to induce expression.

Most growth-regulatory molecules have a relatively short half life in the circulation, as exemplified by PDGF, which has a half life of two minutes. ${ }^{16}$ Binding proteins for many growth factors have been identified in plasma and may be involved in their rapid clearance. $a_{2}-$ Macroglobulin, quantitatively one of the principal components of plasma, has been identified as a binding protein for bFGF, IL-1, IL-2, IL-6, PDGF, and TGF- $\beta .{ }^{19}$ In some, but not all cases, interaction with $\alpha_{2}$ macroglobulin inhibits both binding and activity of the growth-regulatory molecules. Therefore, it has been proposed that the binding proteins may prevent the action of the growth factors at sites distal to the site of their release. Exceptions to rapid clearanceू๊ are the insulin-like growth factors (IGF-P and IGF-II) that circulate in the plasmas associated with binding proteins.

Because growth factors and cytokines are expressed at low levels or sequestered in inactive pools in normal cells, the manner im which their synthesis and release are regu lated represents an important element of con윽 trol. A consistent pattern that has been demonstrated in vitro, and in many cases in vivo, is autocrine induction of growth factor mRNA levels as well as induction by exoge nous growth factors and cytokines. This apparent auto-amplification system is also typified by PDGF $^{16}$ which can induce exprese sion of both PDGF and PDGF recepton genes. Cytokines such as IL-1, TNF- $a$, and TGF- $\beta$ also induce PDGF expression, and the proliferation induced by these cytokines can be almost totally abrogated with anti PDGF antibodies.

GROWTH FACTORS IN ATHEROSCLEROSIS: EVIDENCE FOR ALTERED CONCENTRATIONS AND SPECIFIC ROLES

Several studies have examined the expressio of the various cytokines and growth factor 8 that may be involved in the cellular changes that accompany developing lesions. Increase concentrations of IL- 1 , TNF- $\alpha$, IFN- $\gamma$, an $\bar{\phi}$ PDGF-B chain have been observed in the lesions of atherosclerosis. ${ }^{18}$ PDGF-B chain, a potent smooth muscle chemoattractant and 
mitogen, has been localised in all stages of lesion development ${ }^{20}$ and was seen to be principally associated with macrophages. Expression of the PDGF $\beta$-receptor subunit, specific for the PDGF-B chain, was shown to be increased in developing lesions, particularly in association with inflammatory cells. Interferon- $\gamma$, a secretory product of activated $\mathrm{T}$ cells, was detected in and around some of the lymphocytes in human lesions. ${ }^{6}$ This was associated with a high frequency of HLA-DR and VLA-1 expression and with a much lower frequency of IL-2 expression, similar to that reported in other chronic inflammatory conditions. Thus, there are several examples of a clear association between expression of particular growth factors or cytokine and lesions of atherosclerosis but not the normal artery wall. However, until specific studies of antagonists or antibodies are performed, the relative roles of these growth factors and cytokines in the pathogenesis of the lesions are based on their activities, observed primarily in vitro studies as discussed above.

Role of the local environment in the regulation of smooth muscle cell migration, proliferation, and metabolism The metabolic derangements leading to SMC migration, proliferation, cholesteryl ester deposition, and plaque development are incompletely understood. As the figure and the table show, in addition to growth-regulatory molecules, several products of arterial

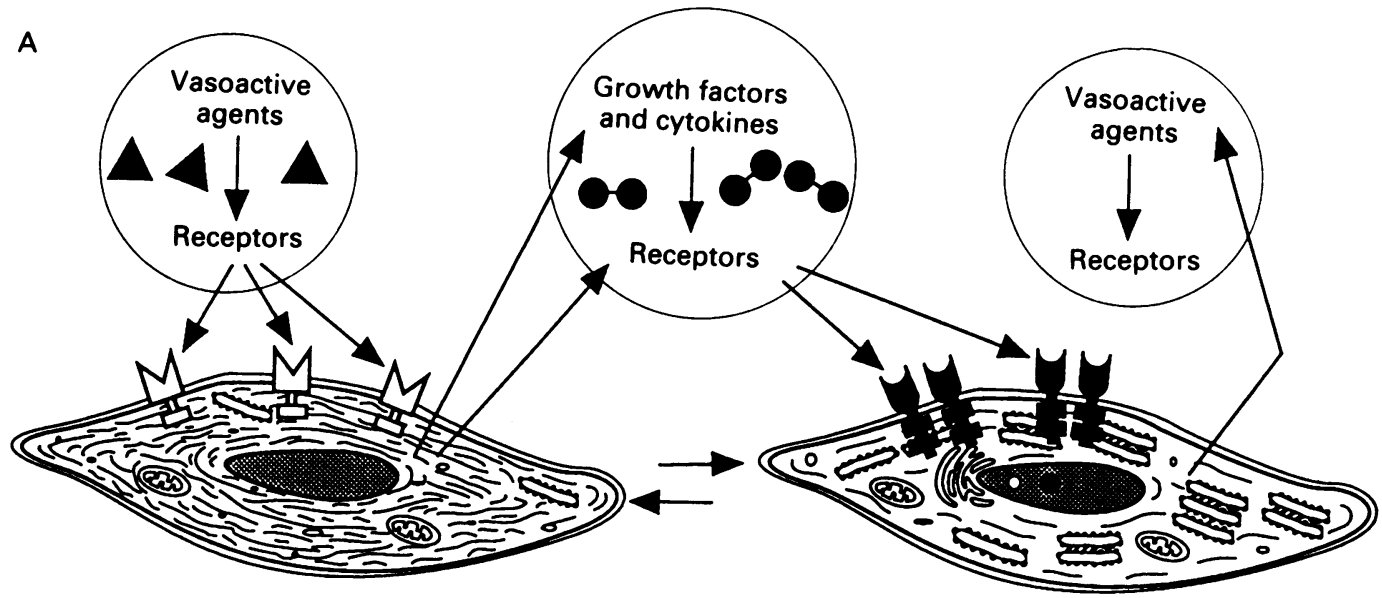

Contractile SMC

Synthetic SMC
Contractile SMC

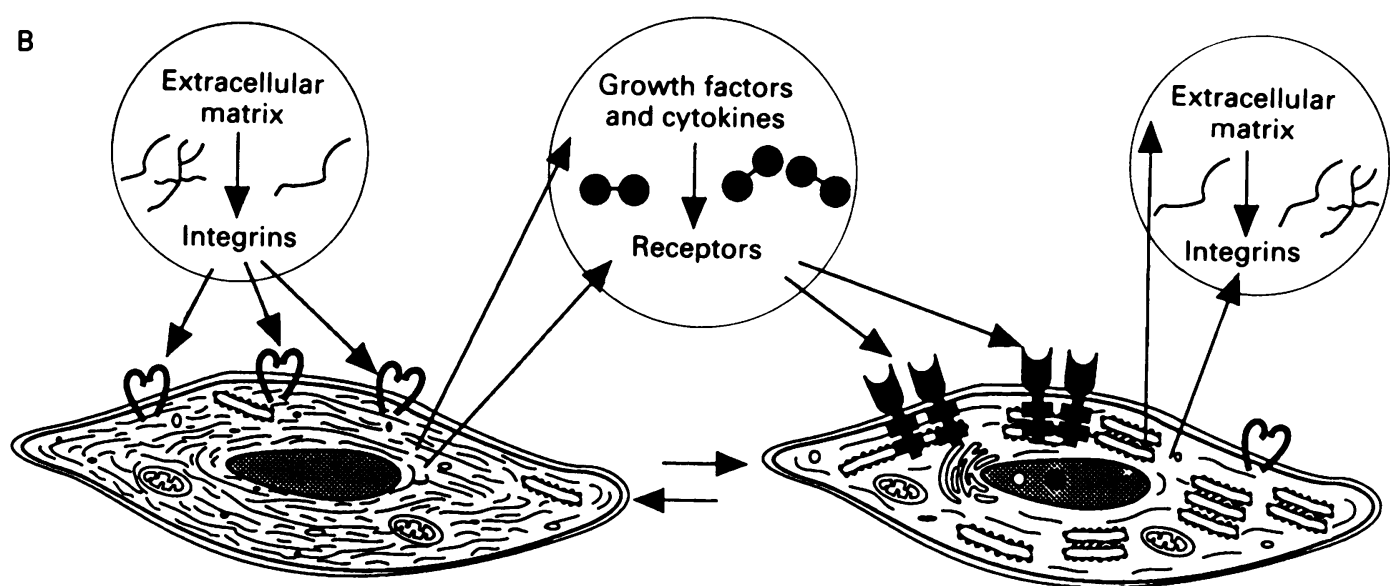

Synthetic SMC

Figure 1 Interplay among the different regulatory mechanisms of smooth muscle cell migration, proliferation, and metabolism. (A) Both growth factors and vasoactive agents (described more completely in the table and the text) can stimulate smooth muscle cell (SMC) migration, proliferation, and altered metabolism. Vasoactive agents are thought to act primarily on "contractile" SMCs. Some of the vasoactive agents maintain cells in the "contractile" state while others promote SMC modulation to the synthetic state. The vasoactive agents may also stimulate migration, proliferation, and altered metabolism. Some of these activities may be mediated by the ability of vasoactive agents to alter the expression of growth factors and their receptors. Thus alterations in the level of vasoactive agents or access of the vasoactive agents to the SMCs may modulate their phenotypic state and function. Growth factors, thought to act primarily on "synthetic" SMCs in which growth factor receptors are upregulated, stimulate SMC migration, proliferation, and altered metabolism including matrix synthesis and lipid accumulation. Growth factors and cytokines are also able to alter the synthesis of the vasoactive agents. Their central role in the cellular changes associated with developing lesions of atherosclerosis make them prime candidates for interventional therapy. (B) The interrelation between growth factors and the extracellular matrix.

The extracellular matrix acting through its cell surface receptors, the integrins, is able to alter SMC phenotype and, in some cases, cell proliferation and migration (table). The extracellular matrix is also able to bind some of the growth factors and in so doing alter growth factor activity. Growth factors and cytokines, acting through their specific cell surface receptors, increase the synthesis of specific matrix constituents and alter the expression of integrins. Thus the interplay between growth factors and the extracellular matrix may be critical in determining SMC function. 
wall cells and infiltrating cells may act on SMCs. Are any of these molecules in some way responsible for the phenotypic changes seen in smooth muscle cells in developing lesions? Or are their altered levels simply a secondary response to changes in the artery wall? What are the initiating factors and what are the reactive changes to these factors?

POSSIBLE ROLE OF VASOACTIVE SUBSTANCES IN ALTERING SMOOTH MUSCLE CELL METABOLISM The idea that contractile agents derived from plasma, nerves, circulating cells, endothelial cells, or SMCs themselves might play a part in mediating SMC phenotype and growth is consistent with changes observed in hypertension and the normal physiological processes in which SMC mass is altered in response to increase in workload. ${ }^{21}$ In some cases, such as SMC stimulation with angiotensin II or arginine vasopressin, there is an increase in cell protein synthesis and cell hypertrophy without stimulation of proliferation. The endothelium is a source of many vasoactive agents and their secretion is critical to the maintenance of normal homoeostasis. These include eicosanoids, products of arachidonic acid (in particular prostacyclin $\mathrm{PGI}_{2}$, and thromboxane $\mathrm{A}_{2}\left(\mathrm{TA}_{2}\right)$ from platelets. ${ }^{22}$ Experimental data demonstrate that eicosanoids derived from the arterial wall maintain the cells in a contractile, quiescent phenotypic state, in which they are responsive to vasoactive stimuli but not to mitogens. In addition, endogenously synthesised eicosanoids help to maintain cellular cholesterol content at a low concentration. This is accomplished by promoting net cellular cholesteryl ester hydrolysis. The capacity of vascular tissue to generate eicosanoids decreases with age, in diabetes mellitus, with smoking, and with viral infections-all major risk factors for atherosclerosis.

Endothelium-derived relaxing factor-nitric oxide (EDRF-NO) is another vasoactive substance whose production may alter SMC function. EDRF-NO relaxes SMCs, and the common release of both EDRF-NO and prostacyclin by increased shear stress suggests that these substances may act in concert to regulate endothelial-platelet interactions as well as vascular tone. ${ }^{23}$ Some data suggest that release of EDRF-NO is impaired in experimentally induced diabetes. Since EDRF-NO can inhibit SMC proliferation, decreased formation of EDRF-NO could contribute to atherosclerosis at several levels. The thickening of the artery wall could also form a barrier limiting the penetration and thus exposure of SMCs to endothelialderived eicosinoids and EDRF-NO.

On the other hand, eicosinoids derived from activated monocytes and neutrophils may augment the inflammatory response by promoting SMC modulation to the synthetic phenotype. 12-Hydroxyeicosatetraenoic acid (12-HETE) (an eicosanoid synthesised by activated platelets) and leukotriene B4 (a neutrophil lipoxygenase product) are both chemoattractants for SMCs. In contrast, prostaglandin $\left(\mathrm{PGE}_{1}\right)$ has been shown to inhibit SMC proliferation while data on SMC proliferation in response to 12-HETE and LTB4 are contradictory. Thus reductions in $\underline{ }$ eicosanoid synthetic capacity may promote cholesterol accumulation in addition to the stimulation of SMCs into a migratory and proliferative state in response to specific eicosanoids and released growth factors. The $\stackrel{\bar{S}}{\rightarrow}$ vasoconstrictor endothelin may also con- $\overline{0}$ tribute to altered SMC activity. Endothelin 흘 has been shown to stimulate SMC prolifera- $\frac{\bar{D}}{\bar{D}}$ tion and is induced by thrombin, insulin, and $\stackrel{\mathbb{Q}}{\varrho}$ TGF- $\beta .^{23}$ Though the function of endothelin $\%$ is not well understood, its expression is $\overrightarrow{0}$ increased locally with platelet release and thrombus formation as well as in diabetic $\vec{\omega}$ patients, and therefore it may contribute to the progression of vascular disease.

Thus several vasoactive agents may play a 8 part in lesion development because of their direct effects on SMC function. However, it is also clear (as suggested in figure A) that $\frac{0}{0}$ growth regulatory molecules are capable of regulating the release of many of these growth $\mathscr{O}$ vasoactive agents. It has also been shown 을 recently that prolonged treatment of cells $\overrightarrow{ }$ with vasopressin will induce desensitisation of the cells to stimulation by PDGF. ${ }^{24}$ What then is the temporal importance and relation between primary and secondary events for $\vec{\theta}$ each of the known risk factors of atheroscle- $\omega$ rosis?

EXTRACELLULAR PRODUCTS AND REGULATION OF SMC FUNCTION

Heparin is a product of endothelial cells and SMCs that may maintain the SMC contractile phenotype and inhibit both SMC migration and proliferation. ${ }^{14}$ This inhibition of proliferation and migration is specific to heparin and is not seen with other glycosaminoglycans. The inhibition by heparin is also specific to SMCs and is not observed with endothelial cells. Heparin can be 3 released by mast cells and endothelial cells or can be released by heparanase from platelets, monocytes, lymphocytes, neutrophils, or 웅 SMCs themselves. Several activities have been attributed to heparin, including sequestration of growth factors, because both $N$ PDGF and BFGF bind tightly to heparin; release of the SMC growth inhibitor TGF- $\beta$ $N$ from a carrier protein by heparin; and alteration of matrix degradation and consequently 2 the ability of the cell to move and divide. Altered matrix composition in response to $\stackrel{\mathbb{D}}{\overparen{D}}$ heparin can also lead to modulation of the SMC phenotype.

Fibronectin and laminin, glycoprotein components of the extracellular matrix, have $\frac{O}{\mathbb{D}}$ different effects on the phenotype of SMCs in culture. ${ }^{12}$ Fibronectin promotes modulation of the cells from a contractile to a synthetic o phenotype, whereas laminin has the opposite effect. Increased amounts of fibronectin have been seen in developing lesions of atherosclerosis. Thrombospondin, another matrix-associated glycoprotein released from platelets and synthesised by activated arterial cells, has 
been shown to promote SMC proliferation. Additionally, it shares common properties with two other matrix-associated glycoproteins, SPARC and tenascin, that have been proposed to modulate cell-matrix interactions. ${ }^{25}$ It has been proposed that these three matrix-associated glycoproteins exert an "anti-adhesive" function that leads to cell rounding and partial detachment from the substratum - a requirement for cell migration and proliferation. These three proteins are present in tissues that are undergoing rapid change in which cell matrix interactions are continually being altered to facilitate tissue growth and repair, including lesions of atherosclerosis.

The alterations in cell shape seen in response to these three "anti-adhesive" matrix glycoproteins are related directly to the number and distribution of focal adhesions on the cell surface. These link the cytoskeleton and its related components to the proteins and proteoglycans of the extracellular matrix through the family of cell adhesion surface receptors-the integrins. ${ }^{26}$ Some integrins also mediate cell-cell adhesion events. Most integrins are expressed on a wide variety of cells, most of which can express several integrins. This diversity allows individual cells to vary their adhesive properties, both in terms of their specificity and affinity, by selective expression of integrins. The cytoplasmic domains of integrins are targets for regulatory events that can alter integrin affinity. It is therefore not surprising that growth-regulatory molecules modulate integrin expression. This is also consistent with the ability of integrins to transmit signals into cells, which would be dependent on the nature of the adjacent cells or extracellular matrix ligands. Occupation of integrin receptors by their ligands leads to tyrosine phosphorylation and cytoplasmic alkalinisation, pathways used by growth-regulatory molecules. Therefore, it has been proposed that integrins and growth-regulatory molecules may act synergistically in stimulating these signals. Figure 1B shows the possible interplay between integrins, extracellular matrix molecules, growth-regulatory molecules, and SMCs.

Though not shown in the figure or listed in the table, lipoproteins deposited in the artery wall and altered by the cells within the artery may also modulate SMC function. The effects of LDL or oxidised LDL on vasoactive agents, extracellular matrix, and growth factor expression in SMCs are largely undefined and require further investigation.

\section{Intervention studies and in vivo} administration : what do they suggest?

A limited number of in vivo studies have been performed in which the consequences of administration of growth factors and cytokine or specific antagonists to these growthregulatory molecules have been examined. Our lack of knowledge of the physiological concentrations of any of these mediators and their alterations in disease processes makes interpretation of these studies difficult. Most in vivo studies have been performed by means of balloon injury of the normal carotid artery of the rat. ${ }^{15}$ Passage of the inflated balloon through the artery removes all of the endothelial cells, and at the same time expansion of the artery injures the SMCs located in the innermost layers of the media. Mural thrombus formation precedes and accompanies proliferation of the innermost medial SMCs within 48 hours of injury. Subsequently, SMCs migrate from the media into the intima and proliferate, leading to a thickened intima consisting of several layers of SMCs which with time become surrounded by connective tissue matrix.

The importance of thrombosis in the development of atherosclerotic lesions ${ }^{23}$ as well as platelets as a source of several SMC mitogens, led to examination of the response to balloon injury in thrombocytopenic animals. ${ }^{15}$ Though antiplatelet treatment had no effect on the replication rate of the medial SMCs at two days, the intimal lesion area seen on day 7 in the thrombocytopenic animals was significantly smaller than that seen in control rats. These studies suggested that platelet products may primarily regulate movement into the intima. Consistent with this hypothesis are the findings in the same model after administration of a neutralising antibody to PDGF, the major SMC mitogen and chemoattractant in platelets. Examination of the arteries eight days after angioplasty showed a $40 \%$ reduction in the neointimal thickness of the lesions in animals treated with anti-PDGF IgG compared with those treated with non-immune IgG. ${ }^{27}$ However, autoradiographic studies showed no differences between days 7 and 8 after injury in the labelling index between the animals treated with non-immune IgG and those treated with anti-PDGF. Thus, the principal effect in this particular study seems to be on the migratory response in which SMCs move from the media into the intima to form the neointimal lesion.

bFGF is also a potent stimulant of SMC proliferation. Though bFGF is not secreted by SMCs or endothelial cells, it is released from injured and dying cells and therefore may contribute to the cellular changes observed after balloon injury. Administration of neutralising anti-FGF antibodies reduced by $80 \%$ the medial SMC proliferation seen at two days. ${ }^{28}$ However, the intimal lesion observed at eight days was not significantly reduced. It remains to be seen whether the ineffectiveness of the antibody to reduce lesion size at day 8 was due to incomplete neutralisation of bFGF after injury or whether growth factors other than bFGF are responsible for SMC migration and intimal proliferation. The factors responsible for the neointimal proliferation that occurs between days 8 and 15 in this model also remain to be determined.

Two SMC growth stimulators, PDGF and bFGF, and two SMC growth inhibitors, 
IFN- $\gamma$ and heparin, and an angiotensin converting enzyme (ACE) inhibitor have also been infused into rats after balloon catheter injury. Continuous infusion of PDGF- BB for seven days after gentle denudation of the rat carotid artery induced a 15 -fold increase in intimal lesion area compared with the animals treated with the vehicle. ${ }^{29}$ The animals were also continuously infused with $\left[{ }^{3} \mathrm{H}\right]-$ thymidine, and analysis of autoradiograms showed a 21-fold increase in the number of unlabelled cells in the neointima of animals treated with PDGF-BB, with no change in the total number of non-dividing cells in the intima plus media. Though thymidine incorporation by medial smooth muscle cells did increase fourfold in the PDGF-treated animals, the principal effect of PDGF-BB on lesion formation was stimulation of medial SMC migration into the intima. Administration of bFGF over an eight hour period after balloon angioplasty of the rat carotid increased replication from $11.5 \%$ in controls to $54.8 \%$ when measured at two days. ${ }^{28}$ However, in unmanipulated cells, SMCs were unresponsive to infused bFGF. Prolonged administration of bFGF for two weeks after balloon injury caused an approximately twofold increase in intimal thickening. Thus both the antibody inhibition studies and infusion experiments with bFGF and PDGF support a role for these two factors in promoting in vivo medial SMC proliferation and migration, respectively.

In contrast, infusion of IFN- $\gamma$, the SMC growth inhibitor produced by $\mathrm{T}$ cells, reduced intimal cross sectional area by $50 \%$ at two weeks after balloon injury and also inhibited $\left[{ }^{3} \mathrm{H}\right]$-thymidine incorporation at two days by $75 \% .^{30}$ The difference in the IFN- $\gamma$ treated animals persisted in rats examined 10 weeks after injury. Inhibition of the neointimal accumulation of SMCs after balloon angioplasty was also seen after administration of heparin ${ }^{15}$ and of inhibitors of angiotensinconverting enzyme (ACE). ${ }^{31}$ Heparin inhibits intimal thickening and blocks early SMC proliferation and migration by altering the composition of the pericellular matrix and by decreasing deposition of elastin and interstitial collagen while increasing deposition of proteoglycans. The action of heparin does not depend on its anticoagulant activity. Heparin also seems to stimulate endothelial regeneration. Although the structural elements on the heparin molecule that are necessary for optimal inhibitory activity have been defined, the mechanism of action of heparin inhibition is not known. Two possibilities have been suggested: (a) that heparin prevents local accumulation of released growth factors such as bFGF or $(b)$, owing to its ability to inhibit tissue-type plasminogen activator and interstitial collagenase activities, heparin may interfere with the ability of the SMC to degrade its surrounding matrix, an event necessary for migration and possibly proliferation. It is also unclear how ACE inhibitors block neointimal thickening, where angiotensin II is generated, and how it acts to stim- ulate SMC proliferation. An important question raised by the ACE inhibitor studies is the variability in response seen in different animal models of restenosis. ${ }^{32}$

Thus in vivo studies of intervention and of administration of growth regulatory mole- $\stackrel{\mathbb{D}}{2}$ cules have helped to identify factors or processes involved in repair of the artery after $\Rightarrow$ injury by balloon angioplasty. However, the cellular makeup, and thus the interaction that? can occur in an occluded artery, is different $\frac{\overline{-}}{\overline{6}}$. from those of a normal artery. Therefore, the $\frac{\bar{D}}{\sigma}$ interrelation (fig 1) between the SMCs, the 2 extracellular matrix, and growth regulatoryo and vasoactive molecules may require differ- $\overrightarrow{0}$ ent interventional approaches in diseased vessels and normal vessels.

\section{Summary}

In this review we have tried to identify the characteristic features of SMCs in developing? lesions of atherosclerosis and the extracellularo factors that may be involved in regulating $\mathcal{c}$ these altered features. Though the list seems long and complex there is probably a great 을 deal of interplay among the different regula- $\vec{c}$ tory mechanisms. The function and activities of SMCs in the artery are dependent on the milieu created by the surrounding cells and $₹$ the components of the extracellular matrix. In $\overrightarrow{0}$ the normal, uninjured media of the artery, $\omega$ SMC phenotype and function seem to be in large part determined by the extracellular matrix in which they are embedded and by diffusible factors, in particular from endothelial cells. Endothelial cell injury, infiltration of $\stackrel{\odot}{\Phi}$ monocytes and lymphocytes, and ultimately, $\overrightarrow{\vec{\partial}}$ thrombosis and platelet release, as seen in $\frac{\circ}{3}$ developing lesions of atherosclerosis, dramatically alter the balance of growth-regulatory and vasoactive factors present in the local environment. These extracellular factors (table and figure) can alter both SMC pheno- $\frac{7}{0}$ type, and thus responsiveness, and SMC migration, proliferation, and synthesis of the $\delta$ extracellular matrix. A better understanding of how specific factors mediate these respons-? es, should make it possible to determine the $D$ ways in which the SMC response can be을 modulated. Though growth regulatory mole- N cules seem to be key to this process, the challenge for the future is to understand their ${ }^{\circ}$ regulation in the environment of the artery $\omega$ wall and the interplay between growth-regula-

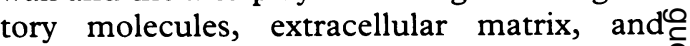
vasoactive agents.

This work was supported in part by National Institute of Health grants HL18645 and HL47151 and an unrestricted orant for cardiovascular research from Bristol-Myers Squibbo Company. We thank Kris Carroll for help in the preparation $\mathbb{Q}$ of the figures.

Stary HC, Blankenhorn DH, Chandler AB, Glagov S, Insull $\mathrm{W}$, Richardson $\mathrm{M}$, et al. A definition of the intimaof human arteries and its atherosclerosis-prone regions.Arteriosclerosis 1992;12:120-34.

2 Ross R. The pathogenesis of atherosclerosis-An update. N Engl ₹ Med 1986;314:488-500.

Munro JM, Cotran RS. The pathogenesis of atherosclerosis: Atherogenesis and inflammation. Lab Invest 1988; 58:249-61.

4 Steinberg D, Parthasarathy S, Carew TE, Khoo JC Witztum JL. Beyond cholesterol: Modifications of low- 
density lipoprotein that increase its atherogenicity. $N$ Engl f Med 1989;320:915-24.

5 Ip JH, Fuster V, Badimon JJ, Taubman MB, Chesebro $\mathrm{JH}$. Syndromes of accelerated atherosclerosis: Role of vascular injury and smooth muscle cell proliferation. $f$ Am Coll Cardiol 1990;15:1667-87.

6 Libby P, Hansson GK. Involvement of the immune system in human atherogenesis:current knowledge and unanswered questions. Lab Invest 1991;64:5-15.

7 Clowes AW, Schwartz SM. Significance of quiescent smooth muscle migration in the injured rat carotid smooth muscle migration in the
artery. Circ Res 1985;56:139-45.

8 Brown BG, Albers JJ, Fisher LD, et al. Regression of coronary artery disease as a result of intensive lipid-lowering therapy in men with high levels of apolipoprotein B. $N$ Engl $\mathcal{F}$ Med 1990;323:1289-98.

9 Gordon D, Reidy MA, Benditt EP, Schwartz SM. Cell proliferation in human coronary arteries. Proc Natl Acad Sci USA 1990;87:4600-4.

10 O'Brien E, Gordon D, Yoon S, Schwartz S, Stewart D. EII proliferation in primary and restenotic human coronary atherosclerosis [abstr]. I Am Coll Cardiol 1992;19: 328.

11 Campbell GR, Campbell JH. The phenotypes of smooth muscle expressed in human atheroma. In: Lee KT, Onodera K, Tanaka $\mathrm{K}$, eds. Atl II: recent progress in Onodera K, Tanaka $\mathrm{K}$, eds. Atl II: recent progress in atherosclerosis research. New

12 Thyberg J, Hedin U, Sjöplund M, Palmberg L, Bottger BA. Regulation of differentiated properties and proliferation or arterial smooth muscle cells. Arteriosclerosis 1990;10:966-90.

13 O'Brien K, Nagano Y, Gown A, Chait A. Probucol treatment affects the cellular composition but not antioxidised low density lipoprotein immunoreactivity of plaques from Watanabe heritable hyperlipidemic rabbits. Arteriosclerosis 1991;11:751-9.

14 Wight TN. Cell biology of arterial proteoglycans. Arteriosclerosis 1989;9:1-20.

15 Clowes AW, Reidy MA. Prevention of stenosis after vascular reconstruction: Pharmacologic control of intimal cular reconstruction: Pharmacologic control of intima
hyperplasia-A review. F Vasc Surg 1991;13:885-91.

16 Raines EW, Bowen-Pope DF, Ross R. Platelet-derived growth factor. In: Sporn MB, Roberts AB, eds. Handbook of experimental pharmacology: peptide growth factors and their receptors I. New York:Springer-Verlag, vol 95, 1990:173-262.

17 Sporn MB, Roberts AB, eds. Handbook of experimental pharmacology: peptide growth factors and their receptors. New York:Springer-Verlag, vol 95, 1990.

18 Raines EW, Ross R. Vascular effects of cytokines and growth-regulatory molecules. In: Gearing A, Kossio J, Oppenheim J, eds. The clinical applications of cytokines: role in pathogenesis, diagnosis and therapy. Oxford: Oxford University Press.

19 LaMarre J, Wollenberg GK, Gonias SL, Hayes MA. Cytokine binding and clearance properties of proteinase-activated $a_{2}$ macroglobulins. Lab Invest 1991; 5:3-14.

20 Ross R, Masuda J, Raines EW, et al. Localisation of PDGF-B protein in macrophages in all phases of atherogenesis. Science 1990;248:1009-12.

21 Owens GK. Control of hypertrophic versus hyperplastic growth of vascular smooth muscle cells. Am f Physiol growth of vascular
$1989 ; 257: 1755-65$.

22 Pomerantz KB, Hajiar DP. Eicosanoids in regulation of arterial smooth muscle cell phenotype, proliferative capacity, and cholesterol metabolism. Arteriosclerosis 1989;9:413-29.

23 Vane JR, Änggard EE, Botting RM. Regulatory functions of the vascular endothelium. $N$ Engl $\mathcal{F}$ Med 1990; 323:27-36.

24 Domin J, Rozengurt E. Heterologous densensitization of Platelet-derived growth factor-mediated arachidonic acid release and prostaglandin synthesis. $f$ Biol Chem 1992;267:15217-23.

25 Sage EH, Bornstein P. Extracellular proteins that modulate cell-matrix interactions: SPARC, tenascin, and thrombospondin. $₹$ Biol Chem 1991:266:14831-34.

26 Hynes RO. Integrins: versatility, modulation, and signalling in cell adhesion. Cell 1992;62:11-25.

27 Ferns GAA, Raines EW, Sprugel KH, Motani AS, Reidy MA, Ross R. Inhibition of neointimal smooth muscle accumulation after angioplasty by an antibody to PDGF. Science 1991;253:1129-32.

28 Reidy MA, Lindner V. Basic FGF and growth of arterial cells. Ann NY Acad Sci 1991;638:290-9.

29 Jawien A, Bowen-Pope DF, Lindner V, Schwartz SM, Clowes AW. Platelet-derived growth factor (PDGF) promotes smooth muscle migration and intimal thickening in a rat model of balloon angioplasty. 7 Clin Invest ing in a rat model

30 Hansson GK, Holm J. Interferon- $\gamma$ inhibits arterial stenosis after injury. Circulation 1991;84:1266-72.

31 Powell JS, Clozel J-P, Muller RKM, Kuhn H, Hefti F, Hosang M, Baumgartner HR. Inhibitors of angiotensinconverting enzyme prevent myointimal proliferation after vascular injury. Science 1989;245:186-8.

32 Ferrell M, Fuster V, Fold HK, Chesebro JH. A dilemma for the 1990s:choosing appropriate experimental animal model for the prevention of restenosis. Circulation 1992;85:1630-1. 